\title{
Análisis y aplicabilidad del score PESI-ECHO en pacientes con tromboembolia pulmonar aguda
}

\section{Analysis and practical utility of PESI-ECHO score in patients with acute pulmonary embolism}

\section{Cristhian E. Scatularo ${ }^{1,2 *}$}

${ }^{1}$ Servicio de Cardiología, Sanatorio de la Trinidad de Palermo; ${ }^{2}$ Servicio de Insuficiencia Cardiaca y Trasplante Cardiaco, Fundación Favaloro. Ciudad Autónoma de Buenos Aires, Argentina

El artículo original The addition of echocardiographic parameters to PESI risk score improves mortality prediction in patients with acute pulmonary embolism: PESI-Echo score, publicado en European Heart Journal por parte de investigadores del Consejo Argentino de Residentes de Cardiología (CONAREC), del cual he tenido el honor de formar parte, es un interesante análisis efectuado a partir de la cohorte del Registro CONAREC XX, que incluyó 684 pacientes internados por tromboembolia pulmonar (TEP) aguda en la República Argentina.

La TEP aguda constituye la tercera causa de muerte cardiovascular más prevalente en Europa y EE.UU., por lo que su diagnóstico precoz y estratificación de riesgo de mortalidad son claves para seleccionar el tratamiento adecuado. La TEP de riesgo bajo presenta una baja mortalidad, por lo que la anticoagulación y egreso hospitalario precoz son las estrategias mas razonables, y por el contrario, la TEP con descompensación hemodinámica (alto riesgo de mortalidad a siete días) requiere la reperfusión pulmonar por cualquier método posible. Sin embargo, la TEP de riesgo intermedio presenta un amplio rango de mortalidad (del 3 al 15\% según diversos reportes), dado que incluye pacientes con múltiples variables heterogéneas de pronóstico adverso y a aquellos con inestabilidad hemodinámica incipiente o múltiples comorbilidades, escenario en donde los estudios no definen claramente el beneficio de la reperfusión por sobre la anticoagulación sola. Debemos tener en cuenta, sin embargo, que dichos estudios han sido efectuados estratificando a los pacientes mediante herramientas validadas que no incluyen algunas variables de riesgo de mortalidad relevantes. En este sentido resulta fundamental contar con herramientas que mejoren dicha estratificación.

El índice de gravedad de embolia pulmonar (PESI) es un modelo de predicción de riesgo de muerte por TEP aguda ampliamente validado y que solamente utiliza parámetros clínicos para estratificar a los pacientes en cinco niveles de riesgo, con un área bajo la curva ROC de hasta 0.79 según diferentes estudios de validación externa.

Por otra parte, el ecocardiograma transtorácico (ETT) es fundamental en la estratificación de riesgo de estos pacientes para el estudio de la función del ventrículo derecho (VD) y la presión sistólica de la arteria pulmonar (PSAP). Pese a que ningún parámetro del ETT es considerado el gold standard para la estratificación de estos pacientes, la excursión sistólica del plano anular tricuspídeo (TAPSE) como determinante de contractilidad del VD y la PSAP son los que presentan mayor capacidad discriminativa como variable continua.

\section{Correspondencia:}

${ }^{*}$ Cristhian E. Scatularo

E-mail: emmanuelscatularo@gmail.com DOI: 10.24875/ACM.M21000079
Fecha de recepción: 1-08-2021

Fecha de aceptación: 18-08-2021
Disponible en internet: 29-10-2021 Arch Cardiol Mex. 2021;91(4):394-395 www.archivoscardiologia.com CC BY-NC-ND (http://creativecommons.org/licenses/by-nc-nd/4.0/). 
En nuestro estudio presentamos un nuevo score, denominado PESI-ECHO, que combinó estas variables $y$ fue determinado mediante la siguiente ecuación (PESI-ECHO = PESI + PSAP - TAPSE). EI área bajo la curva ROC fue de 0.75 para el score PESI y 0.82 para el PESI-ECHO, diferencia estadísticamente significativa a favor del nuevo modelo, con único punto de corte de predicción de 128 puntos (sensibilidad $82 \%$, especificidad $69 \%$, valor predictivo negativo $96 \%$ y valor predictivo positivo $27 \%$.). De esta manera, hemos demostrado que el score PESI-ECHO tiene una mejor capacidad para estratificar a los pacientes con TEP aguda que el PESI. Además, su utilización no presenta mayor complejidad, dada la habitual utilización del ETT y la simplicidad de la medición del TAPSE y la PSAP.

Al aplicar el score PESI-ECHO a la cohorte global del Registro CONAREC XX, se demostró un índice neto de reclasificación del $9.8 \%$, lo que demuestra que aproximadamente el $10 \%$ de los pacientes han sido incorrectamente estratificados con el score PESI, impactando seriamente en la elección del tratamiento y la evolución posterior.

Desde un análisis personal, y aunque aún no ha sido realizada su validación externa, sostengo que los futuros ensayos clínicos aleatorizados y estudios observacionales de pacientes con TEP aguda podrían utilizar esta nueva herramienta a fin de lograr una correcta estratificación de los pacientes y obtener información fidedigna en el análisis de los puntos finales intrahospitalarios. Desde la práctica asistencial, el score PESI-ECHO resulta una herramienta de fácil aplicación en la estratificación de riesgo de pacientes con TEP aguda sin descompensación hemodinámica y que impactará a la hora de tomar la decisión (muchas veces compleja y difícil) de la reperfusión pulmonar. 